\title{
The Electroweak Model based on the Nonlinearly Realized Gauge Group
}

\section{Daniele Bettinelli}

Albert-Ludwigs Universität Freiburg

E-mail: daniele.bettinelliaphysik.uni-freiburg.de

\section{Ruggero Ferrari}

CTP-MIT, Cambridge, MA

and Università degli Studi di Milano \& INFN, Sez. di Milano

E-mail: ruggero.ferrari@mi.infn.it

\section{Andrea Quadri*}

Albert-Ludwigs Universität Freiburg

and Università degli Studi di Milano \& INFN, Sez. di Milano

E-mail: andrea.quadri@mi.infn.it

\begin{abstract}
A consistent strategy for the subtraction of the divergences in the nonlinearly realized Electroweak Model in the loop expansion is presented. No Higgs field is present in the perturbative spectrum. The local functional equation (LFE), encoding the invariance of the SU(2) Haar measure under local left SU(2) transformations, the Slavnov-Taylor identity, required in order to fulfill physical unitarity, and the Landau gauge equation hold in the nonlinearly realized theory. The quantization is performed in the Landau gauge for the sake of simplicity and elegance. The constraints on the admissible interactions arising from the Weak Power-Counting (WPC) are discussed. The same symmetric pattern of the couplings as in the Standard Model is shown to arise, as a consequence of the symmetries and the WPC. However, two independent mass invariants in the vector meson sector are possible. Therefore the tree-level Weinberg relation is not fulfilled in the nonlinearly realized Electroweak model.
\end{abstract}

International Workshop on QCD Green Functions, Confinement, and Phenomenology - QCD-TNT09 September 07 - 112009

ECT Trento, Italy

\footnotetext{
* Speaker.
} 


\section{Introduction}

A consistent strategy for the subtraction of nonlinearly realized gauge theories order by order in the loop expansion has been recently proposed in [1]-[7]. The discovery of the Local Functional Equation [14], encoding the invariance of the SU(2) path-integral Haar measure under local left $\mathrm{SU}(2)$ transformations, has provided a key tool in order to tame the divergences of this class of theories. The LFE uniquely fixes the dependence of the 1-PI amplitudes involving at least one Goldstone field (descendant amplitudes) in terms of 1-PI amplitudes with no external Goldstone legs (ancestor amplitudes). This establishes a very powerful hierarchy among 1-PI Green functions. While there is an infinite number of divergent descendant amplitudes already at one loop level, only a finite number of ancestor amplitudes exists order by order in the loop expansion if the Weak Power-Counting (WPC) condition is fulfilled [2], [3], [7], [11]. In addition to the LFE, the SlavnovTaylor (ST) identity must be imposed in order to fulfill the requirement of Physical Unitarity [13]. It should be noted that the ST identity does not yield a hierarchy among 1-PI Green functions [7]. Thus the LFE provides an essential tool in order to carry out the consistent subtraction of nonlinearly realized gauge theories. A summary of the formal developments has been given in R.Ferrari's talk [8].

The WPC poses stringent constraints on the admissible terms in the tree-level vertex functional. In order to work out these constraints a convenient strategy is first to perform an invertible change of variables from the original ones to their corresponding $\mathrm{SU}(2)$ gauge-invariant counterparts (bleached variables). Since the bleached variables are SU(2)-invariant, the hypercharge generator coincides on them with the electric charge. Therefore any electrically neutral local monomial depending on the bleached variables and covariant derivatives w.r.t. the U(1) gauge connection $B_{\mu}$ is allowed on symmetry grounds. The requirement of the validity of the WPC imposes a set of constraints among these monomials. It turns out that all the symmetric interactions between ancestor amplitudes present in the Standard Model are recovered [2], [3] by imposing the symmetries and the WPC (no anomalous couplings are allowed). On the other hand, two independent mass invariants are possible in the vector meson sector. Therefore the tree-level Weinberg relation between the $Z$ and $W$ mass does not hold in the nonlinearly realized theory.

\section{Bleached Variables}

The field content of the electroweak model based on the nonlinearly realized $S U(2)_{L} \otimes U(1)$ gauge group includes (leaving aside the ghosts and the Nakanishi-Lautrup fields) the $S U(2)_{L}$ connection $A_{\mu}=A_{a \mu} \frac{\tau_{a}}{2}$ ( $\tau_{a}, a=1,2,3$ are the Pauli matrices), the $U(1)$ connection $B_{\mu}$, the fermionic left doublets collectively denoted by $L$ and the right singlets, i.e.

$$
\begin{gathered}
L \in\left\{\left(\begin{array}{c}
l_{L j}^{u} \\
l_{L j}^{d}
\end{array}\right),\left(\begin{array}{c}
q_{L j}^{u} \\
V_{j k} q_{L k}^{d}
\end{array}\right), \quad j, k=1,2,3\right\}, \\
R \in\left\{\left(\begin{array}{c}
l_{R j}^{u} \\
l_{R j}^{d}
\end{array}\right),\left(\begin{array}{c}
q_{R j}^{u} \\
q_{R j}^{d}
\end{array}\right), \quad j=1,2,3\right\} .
\end{gathered}
$$

In the above equation the quark fields $\left(q_{j}^{u}, j=1,2,3\right)=(u, c, t)$ and $\left(q_{j}^{d}, j=1,2,3\right)=(d, s, b)$ are taken to be the mass eigenstates in the tree-level lagrangian; $V_{j k}$ is the CKM matrix. Similarly we 
use for the leptons the notation $\left(l_{j}^{u}, j=1,2,3\right)=\left(v_{e}, v_{\mu}, v_{\tau}\right)$ and $\left(l_{j}^{d}, j=1,2,3\right)=(e, \mu, \tau)$. The single left doublets are denoted by $L_{j}^{l}, j=1,2,3$ for the leptons, $L_{j}^{q}, j=1,2,3$ for the quarks. Color indexes are not displayed.

One also introduces the $S U(2)$ matrix $\Omega$

$$
\Omega=\frac{1}{v}\left(\phi_{0}+i \phi_{a} \tau_{a}\right), \quad \Omega^{\dagger} \Omega=1 \Rightarrow \phi_{0}^{2}+\phi_{a}^{2}=v^{2} .
$$

The mass scale $v$ gives $\phi$ the canonical dimension at $D=4$. We fix the direction of Spontaneous Symmetry Breaking by imposing the tree-level constraint

$$
\phi_{0}=\sqrt{v^{2}-\phi_{a}^{2}} .
$$

The condition $\langle\Omega\rangle=1$ cannot be imposed at a generic order of perturbation theory.

The $S U(2)$ flat connection is defined by

$$
F_{\mu}=i \Omega \partial_{\mu} \Omega^{\dagger}
$$

The transformation properties under the local $S U(2)_{L}$ transformations are ( $g$ is the $S U(2)_{L}$ coupling constant)

$$
\begin{array}{ll}
\Omega^{\prime}=U \Omega, & B_{\mu}^{\prime}=B_{\mu}, \\
A_{\mu}^{\prime}=U A_{\mu} U^{\dagger}+\frac{i}{g} U \partial_{\mu} U^{\dagger}, & L^{\prime}=U L, \\
F_{\mu}^{\prime}=U F_{\mu} U^{\dagger}+i U \partial_{\mu} U^{\dagger}, & R^{\prime}=R .
\end{array}
$$

Under local $U(1)_{R}$ transformations one has

$$
\begin{array}{ll}
\Omega^{\prime}=\Omega V^{\dagger}, & B_{\mu}^{\prime}=B_{\mu}+\frac{1}{g^{\prime}} \partial_{\mu} \alpha, \\
A_{\mu}^{\prime}=A_{\mu}, & L^{\prime}=\exp \left(i \frac{\alpha}{2} Y_{L}\right) L, \\
F_{\mu}^{\prime}=F_{\mu}+i \Omega V^{\dagger} \partial_{\mu} V \Omega, & R^{\prime}=\exp \left(i \frac{\alpha}{2}\left(Y_{L}+\tau_{3}\right)\right) R .
\end{array}
$$

where $V(\alpha)=\exp \left(i \alpha \frac{\tau_{3}}{2}\right)$.

The electric charge is defined according to the Gell-Mann-Nishijima relation

$$
Q=I_{3}+Y
$$

where the hypercharge operator $Y$ is the generator of the $U(1)_{R}$ transformations (2.6) and $I_{3}$ is an abstract object. The introduction of the matrix $\Omega$ allows to perform an invertible change of variables from the original set of fields to a new set of $S U(2)_{L}$-invariant ones (bleaching procedure). For that purpose we define

$$
\begin{aligned}
& w_{\mu}=w_{a \mu} \frac{\tau_{a}}{2}=g \Omega^{\dagger} A_{\mu} \Omega-g^{\prime} B_{\mu} \frac{\tau_{3}}{2}+i \Omega^{\dagger} \partial_{\mu} \Omega, \\
& \tilde{L}=\Omega^{\dagger} L .
\end{aligned}
$$

Both $w_{\mu}$ and $\tilde{L}$ are $S U(2)_{L}$-invariant, while under $U(1)_{R}$ they transform as

$$
w_{\mu}^{\prime}=V w_{\mu} V^{\dagger}, \quad \tilde{L}^{\prime}=\exp \left(i \frac{\alpha}{2}\left(\tau_{3}+Y_{L}\right)\right) \tilde{L} .
$$

I.e. the electric charge coincides with the hypercharge on the bleached fields, as it is apparent from the comparison of eqs.(2.6), (2.7) and (2.9). 


\section{The Weak Power-Counting}

Any Lorentz-invariant electrically neutral local monomial built out of the components of $w_{a \mu}, \tilde{L}, R$ and covariant derivatives w.r.t. $B_{\mu}$ is allowed on symmetry grounds. We further impose the validity of the WPC condition, i.e. the superficial degree of divergence of any 1-PI graph $\mathscr{G}$ with $N_{A}$ gauge boson external legs and $N_{F}$ fermionic legs must be bounded by

$$
d(\mathscr{G})=(D-2) n+2-N_{A}-N_{F},
$$

where $D$ is the space-time dimension and $n$ is the number of loops. Several comments are in order here. First of all in $D=4$ we see that the number of ancestor divergent amplitudes compatible with the bound (3.1) increases with the loop order $n$. Therefore we refer to formula (3.1) as the weak power-counting condition. Moreover from eq.(3.1) one also sees that the number of divergent ancestor amplitudes is finite at every loop order. We also notice that the UV dimension of the fermion fields in one in the nonlinearly realized theory (instead of $3 / 2$ as in the linearly realized models). The reason is that the gauge-invariant mass terms $l_{R j}^{d} \tilde{l}_{j}^{d}$ generate upon expansion in powers of the Goldstone fields a quadrilinear vertex of the form $l_{R j}^{d} \phi^{2} l_{j}^{d}$ which contains two Goldstone fields. Therefore at one loop level there are logarithmically divergent graphs with four fermionic external legs [2], [3] which limit the UV dimension of massive fermions to 1.

In the absence of massive neutrinos the WPC condition in eq.(3.1) selects a unique classical action given by

$$
\begin{aligned}
& S=\Lambda^{(D-4)} \int d^{D} x\left(2 \operatorname{Tr}\left\{-\frac{1}{4} G_{\mu \nu} G^{\mu v}-\frac{1}{4} F_{\mu v} F^{\mu v}\right\}\right. \\
& +M^{2} \operatorname{Tr}\left\{\left(g A_{\mu}-\frac{g^{\prime}}{2} \Omega \tau_{3} B_{\mu} \Omega^{\dagger}-F_{\mu}\right)^{2}\right\} \\
& +M^{2} \frac{\kappa}{2}\left(\operatorname{Tr}\left\{\left(g A_{\mu}-\frac{g^{\prime}}{2} \Omega \tau_{3} B_{\mu} \Omega^{\dagger}-F_{\mu}\right) \tau_{3}\right\}\right)^{2} \\
& +\sum_{L}\left[\bar{L}\left(i \not \partial+g \not A+\frac{g^{\prime}}{2} Y_{L} \not B\right) L\right]+\sum_{R}\left[\bar{R}\left(i \not \partial+\frac{g^{\prime}}{2}\left(Y_{L}+\tau_{3}\right) \not B\right) R\right] \\
& +\sum_{j}\left[m_{l_{j}} \bar{R}_{j}^{l} \frac{1-\tau_{3}}{2} \Omega^{\dagger} L_{j}^{l}-m_{q_{j}^{u}} \bar{R}_{j}^{q} \frac{1+\tau_{3}}{2} \Omega^{\dagger} L_{j}^{q}\right. \\
& \left.\left.+m_{q_{k}^{d}} V_{k j}^{\dagger} \bar{R}_{k}^{q} \frac{1-\tau_{3}}{2} \Omega^{\dagger} L_{j}^{q}+h . c .\right]\right) .
\end{aligned}
$$

In $D$ dimensions the doublets $L$ and $R$ obey

$$
\gamma_{D} L=-L \quad \gamma_{D} R=R
$$

being $\gamma_{D}$ a gamma matrix that anti-commutes with every other $\gamma^{\mu}$.

In eq.(3.2) we have introduced the charged combinations

$$
w_{\mu}^{ \pm}=\frac{1}{\sqrt{2}}\left(w_{1 \mu} \mp i w_{2 \mu}\right)
$$


with $U(1)$ transformation properties

$$
w_{\mu}^{ \pm^{\prime}}=\exp ( \pm i \alpha) w_{\mu}^{ \pm}
$$

The neutral component $w_{3 \mu}$ is invariant. Therefore one obtains two independent mass terms which can be parameterized as

$$
M^{2}\left(w^{+} w^{-}+\frac{1}{2} w_{3}^{2}\right), \quad \frac{M^{2} \kappa}{2} w_{3}^{2}
$$

Two mass invariants are expected for the vector mesons, as a consequence of the breaking of the global $S U(2)_{R}$ invariance induced by the hypercharge. We remark that in the SU(2) nonlinearly realized Yang-Mills theory the WPC plus the gauge symmetry were compatible with any bilinear term with no derivatives in the bleached gauge field. The unique diagonal Stückelberg mass term was recovered by imposing an additional $S U(2)_{R}$ global symmetry.

\section{Gauge-fixing and External sources}

We adopt a background Landau gauge-fixing in order to preserve the invariance under local $S U(2)_{L}$ transformations. For the purpose we introduce a background gauge field $V_{a \mu}$. The ghosts associated with the $S U(2)_{L}$ symmetry are denoted by $c_{a}$. Their anti-ghosts are denoted by $\bar{c}_{a}$, the Nakanishi-Lautrup fields by $b_{a}$. It is also useful to adopt the matrix notation $c=c_{a} \frac{\tau_{a}}{2}, b=$ $b_{a} \frac{\tau_{a}}{2}, \bar{c}=\bar{c}_{a} \frac{\tau_{a}}{2}$. The abelian ghost is $c_{0}$, the abelian anti-ghost $\bar{c}_{0}$ and the abelian Nakanishi-Lautrup field $b_{0}$. The BRST partner of $V_{a \mu}$ is denoted by $\Theta_{a \mu}$.

We include in the tree-level vertex functional the anti-fields for the $S U(2)_{L}$ BRST transformation (those for the $U(1)_{R}$ BRST transformation are not required since the Abelian ghost is free in the Landau gauge). Thus we set

$$
\begin{aligned}
\Gamma_{\mathrm{GF}}^{(0)}= & \Lambda^{(D-4)} \int d^{D} x\left(b_{0} \partial_{\mu} B^{\mu}-\bar{c}_{0} \square c_{0}+2 \operatorname{Tr}\left\{b \partial_{\mu} A^{\mu}-\bar{c} \partial^{\mu} D[A]_{\mu} c\right.\right. \\
& \left.+V^{\mu}\left(D[A]_{\mu} b-i g \bar{c} D[A]_{\mu} c-i g\left(D[A]_{\mu} c\right) \bar{c}\right)+\Theta^{\mu} D[A]_{\mu} \bar{c}\right\}+K_{0} \phi_{0} \\
& \left.+A_{a \mu}^{*} \mathfrak{s} A_{a}^{\mu}+\phi_{0}^{*} \mathfrak{s} \phi_{0}+\phi_{a}^{*} \mathfrak{s} \phi_{a}+c_{a}^{*} \mathfrak{s} c_{a}+\sum_{L}\left(L^{*} \mathfrak{s} L+\bar{L}^{*} \mathfrak{s} \bar{L}\right)\right)
\end{aligned}
$$

In the above equation $K_{0}$ is the source coupled to the solution of the nonlinear constraint (2.3). The full tree-level vertex functional is finally

$$
\Gamma^{(0)}=S+\Gamma_{G F}^{(0)} .
$$

The introduction of the external sources in eq.(4.1) allows to provide a functional formulation of the relevant identities of the theory ${ }^{1}$

\footnotetext{
${ }^{1} \Gamma_{\varphi}$ denotes the functional derivative of $\Gamma$ w.r.t. $\varphi$
} 
- the LFE (the $x$-dependence is understood)

$$
\begin{aligned}
& (\mathscr{W} \Gamma)_{a} \equiv-\frac{1}{g} \partial_{\mu} \Gamma_{V_{a \mu}}+\varepsilon_{a b c} V_{c \mu} \Gamma_{V_{b \mu}}-\frac{1}{g} \partial_{\mu} \Gamma_{A_{a \mu}} \\
& +\varepsilon_{a b c} A_{c \mu} \Gamma_{A_{b \mu}}+\varepsilon_{a b c} b_{c} \Gamma_{b_{b}}+\frac{\Lambda^{(D-4)}}{2} K_{0} \phi_{a}+\frac{1}{2 \Lambda^{(D-4)}} \Gamma_{K_{0}} \Gamma_{\phi_{a}} \\
& +\frac{1}{2} \varepsilon_{a b c} \phi_{c} \Gamma_{\phi_{b}}+\varepsilon_{a b c} \bar{c}_{c} \Gamma_{\bar{c}_{b}}+\varepsilon_{a b c} c_{c} \Gamma_{c_{b}} \\
& +\frac{i}{2} \tau_{a} L \Gamma_{L}-\frac{i}{2} \bar{L} \tau_{a} \Gamma_{\bar{L}}-\frac{i}{2} L^{*} \tau_{a} \Gamma_{L^{*}}+\frac{i}{2} \tau_{a} \bar{L}^{*} \Gamma_{\bar{L}^{*}} \\
& +\varepsilon_{a b c} \Theta_{c \mu} \Gamma_{\Theta_{b \mu}}+\varepsilon_{a b c} A_{c \mu}^{*} \Gamma_{A_{b \mu}^{*}}+\varepsilon_{a b c} c_{c}^{*} \Gamma_{c_{b}^{*}}-\frac{1}{2} \phi_{0}^{*} \Gamma_{\phi_{a}^{*}} \\
& +\frac{1}{2} \varepsilon_{a b c} \phi_{c}^{*} \Gamma_{\phi_{b}^{*}}+\frac{1}{2} \phi_{a}^{*} \Gamma_{\phi_{0}^{*}}=0
\end{aligned}
$$

The nonlinearity of the realization of the $S U(2)_{L}$ gauge group is revealed by the presence of the bilinear term $\Gamma_{K_{0}} \Gamma_{\phi_{a}}$.

- the ST identity

$$
\begin{aligned}
\mathscr{S} \Gamma & \equiv \int d^{D} x\left[\Lambda ^ { - ( D - 4 ) } \left(\Gamma_{A_{a \mu}^{*}} \Gamma_{A_{a}^{\mu}}+\Gamma_{\phi_{a}^{*}} \Gamma_{\phi_{a}}+\Gamma_{c_{a}^{*}} \Gamma_{c_{a}}\right.\right. \\
& \left.\left.+\Gamma_{L^{*}} \Gamma_{L}+\Gamma_{\bar{L}^{*}} \Gamma_{\bar{L}}\right)+b_{a} \Gamma_{\bar{c}_{a}}+\Theta_{a \mu} \Gamma_{V_{a \mu}}-K_{0} \Gamma_{\phi_{0}^{*}}\right]=0 .
\end{aligned}
$$

- the Abelian ST identity

$$
\begin{aligned}
- & \frac{2}{g^{\prime}} \Lambda^{(D-4)} \square b_{0}-\frac{2}{g^{\prime}} \partial^{\mu} \frac{\delta \Gamma}{\delta B^{\mu}}-\Lambda^{(D-4)} \phi_{3} K_{0}+\phi_{2} \frac{\delta \Gamma}{\delta \phi_{1}}-\phi_{1} \frac{\delta \Gamma}{\delta \phi_{2}}-\frac{1}{\Lambda^{(D-4)}} \frac{\delta \Gamma}{\delta K_{0}} \frac{\delta \Gamma}{\delta \phi_{3}} \\
& -\phi_{3}^{*} \frac{\delta \Gamma}{\delta \phi_{0}^{*}}+\phi_{2}^{*} \frac{\delta \Gamma}{\delta \phi_{1}^{*}}-\phi_{1}^{*} \frac{\delta \Gamma}{\delta \phi_{2}^{*}}+\phi_{0}^{*} \frac{\delta \Gamma}{\delta \phi_{3}^{*}} \\
& +i Y_{L} L \frac{\delta \Gamma}{\delta L}-i Y_{L} \bar{L} \frac{\delta \Gamma}{\delta \bar{L}}+i\left(Y_{L}+\tau_{3}\right) R \frac{\delta \Gamma}{\delta R}-i \bar{R}\left(Y_{L}+\tau_{3}\right) \frac{\delta \Gamma}{\delta \bar{R}} \\
& -i Y_{L} L^{*} \frac{\delta \Gamma}{\delta L^{*}}+i Y_{L} \bar{L}^{*} \frac{\delta \Gamma}{\delta \bar{L}^{*}}=0 .
\end{aligned}
$$

- the Landau Gauge Equation

$$
\Gamma_{b_{a}}=\Lambda^{(D-4)}\left(D^{\mu}[V]\left(A_{\mu}-V_{\mu}\right)\right)_{a}
$$

which implies the ghost equation

$$
\Gamma_{\bar{c}_{a}}=\left(-D_{\mu}[V] \Gamma_{A_{\mu}^{*}}+\Lambda^{(D-4)} D_{\mu}[A] \Theta^{\mu}\right)_{a},
$$

by using the STI (4.4).

Moreover the WPC condition (3.1) can be extended to the full set of fields and external sources [2], [3]. It can be proven that eq.(3.1) holds to all loop orders [2], [3]. 
Despite the fact that both $S U(2)$ and $U(1)_{R}$ are nonlinearly realized, the Ward identity for the electric charge is linear

$$
\begin{aligned}
& -\frac{1}{g^{\prime}} \Lambda^{(D-4)} \square b_{0}+\left(-\frac{1}{g^{\prime}} \partial^{\mu} \frac{\delta}{\delta B^{\mu}}-\frac{1}{g} \partial_{\mu} \frac{\delta}{\delta A_{3 \mu}}-\frac{1}{g} \partial_{\mu} \frac{\delta}{\delta V_{3 \mu}}\right. \\
& +A_{2 \mu} \frac{\delta}{\delta A_{1 \mu}}-A_{1 \mu} \frac{\delta}{\delta A_{2 \mu}}+i Q L \frac{\delta}{\delta L}-i \bar{L} Q \frac{\delta}{\delta \bar{L}}+i Q R \frac{\delta}{\delta R}-i \bar{R} Q \frac{\delta}{\delta \bar{R}} \\
& +\phi_{2} \frac{\delta}{\delta \phi_{1}}-\phi_{1} \frac{\delta}{\delta \phi_{2}}+b_{2} \frac{\delta}{\delta b_{1}}-b_{1} \frac{\delta}{\delta b_{2}}+c_{2} \frac{\delta}{\delta c_{1}}-c_{1} \frac{\delta}{\delta c_{2}} \\
& +\bar{c}_{2} \frac{\delta}{\delta \bar{c}_{1}}-\bar{c}_{1} \frac{\delta}{\delta \bar{c}_{2}}+V_{2 \mu} \frac{\delta}{\delta V_{1 \mu}}-V_{1 \mu} \frac{\delta}{\delta V_{2 \mu}}+\Theta_{2 \mu} \frac{\delta}{\delta \Theta_{1 \mu}}-\Theta_{1 \mu} \frac{\delta}{\delta \Theta_{2 \mu}} \\
& +A_{2 \mu}^{*} \frac{\delta}{\delta A_{1 \mu}^{*}}-A_{1 \mu}^{*} \frac{\delta}{\delta A_{2 \mu}^{*}}+\phi_{2}^{*} \frac{\delta}{\delta \phi_{1}^{*}}-\phi_{1}^{*} \frac{\delta}{\delta \phi_{2}^{*}}+c_{2}^{*} \frac{\delta}{\delta c_{1}^{*}}-c_{1}^{*} \frac{\delta}{\delta c_{2}^{*}} \\
& \left.-i Q L^{*} \frac{\delta}{\delta L^{*}}+i \bar{L}^{*} Q \frac{\delta}{\delta \bar{L}^{*}}\right) \Gamma=0 .
\end{aligned}
$$

The consequences of eq.(4.8) and of the ST identities on the decoupling of the unphysical modes in the neutral sector have been discussed in [2].

\section{The subtraction procedure}

The perturbative expansion is carried out order by order in the number of loops. The LFE is solved by extending the bleaching technique to the full set of ghost fields and external sources [2],[3]. The ST identities can be recursively studied order by order in the loop expansion. The constraints on the divergent ancestor amplitudes are derived by exploiting the nilpotency of the linearized ST operator $\mathscr{S}_{0}$. One has then to solve a cohomological problem in the space of bleached variables of finite dimension, due to the WPC condition [9].

Finite higher order symmetric renormalization, allowed by the WPC and the symmetries of the theory, cannot be reinserted back into the tree-level vertex functional without violating either the symmetries or the WPC. This fact implies that they cannot be interpreted as additional bona fide physical parameters [10] (unlike in the chiral effective field theories approach). We adopt the following Ansatz: minimal subtraction of properly normalized $n$-loop amplitudes

$$
\frac{1}{\Lambda^{D-4}} \Gamma^{(n)}
$$

around $D=4$ should be performed. This subtraction procedure is symmetric [2], [3], [10]. In this scheme the $\gamma_{5}$ problem is treated in a pragmatic approach. The matrix $\gamma_{5}$ is replaced by a new $\gamma_{D}$ which anti-commutes with every $\gamma_{\mu}$. No statement is made on the analytical properties of the traces involving $\gamma_{D}$. Since the theory is not anomalous such traces never meet poles in $D-4$ and therefore we can evaluate at the end the traces at $D=4$.

In this subtraction scheme the dependence on the scale $\Lambda$ cannot be removed by a shift of the tree-level parameters. Hence it must be considered as an additional physical parameter setting the scale of the radiative corrections. 


\section{Conclusions}

The electroweak model based on the nonlinearly realized $S U(2) \otimes U(1)$ gauge group can be consistently defined in the perturbative loop-wise expansion. In this formulation there is no Higgs in the perturbative series.

The present approach is based on the LFE and the WPC. There is a unique classical action giving rise to Feynman rules compatible with the WPC condition. In particular the anomalous couplings, which would be otherwise allowed on symmetry grounds, are excluded by the WPC. Two gauge bosons mass invariants are compatible with the WPC and the symmetries. Thus the tree-level Weinberg relation is not working in the nonlinear framework.

The discovery of the LFE suggests a unique Ansatz for the subtraction procedure which is symmetric, i.e it respects all the identities of the theory. A linear Ward identity exists for the electric charge (despite the nonlinear realization of the gauge group). The strategy does not alter the number of tree-level parameters apart from a common mass scale of the radiative corrections.

The theoretical and phenomenological consequences of this scenario are rather intriguing. An Higgs boson could emerge as a non-perturbative mechanism, but then its physical parameters are not constrained by the radiative corrections of the low energy electroweak processes. Otherwise the energy scale for the radiative corrections $\Lambda$ is a manifestation of some other high-energy physics.

Many aspects remain to be further studied. We only mention some of them here. The issue of unitarity at large energy (violation of Froissart bound) at fixed order in perturbation theory when the Higgs field is removed can provide additional insight in the role of the mass scale $\Lambda$. The electroweak model based on the nonlinearly realized gauge group satisfies Physical Unitarity as a consequence of the validity of the Slavnov-Taylor identity. Therefore violation of the Froissart bound can only occur in evaluating cross sections at finite order in perturbation theory. This requires the evaluation of a scale at each order where unitarity at large energy is substantially violated.

The phenomenological implications of the nonlinear theory in the electroweak precision fit have to be investigated. The 't Hooft gauge derived for the nonlinearly realized $S U(2)$ massive Yang-Mills theory should be extended to the $S U(2) \otimes U(1)$ nonlinearly realized model [5].

Finally the extension of the present approach to larger gauge groups (as in Grand-Unified models) could help in understanding the nonlinearly realized spontaneous symmetry breaking mechanism (selection of the identity as the preferred direction in the $S U(2)$ manifold) and the associated appearance of two independent gauge bosons mass invariants.

\section{References}

[1] D. Bettinelli, R. Ferrari and A. Quadri, Phys. Rev. D 79 (2009) 125028 [arXiv:0903.0281 [hep-th]].

[2] D. Bettinelli, R. Ferrari and A. Quadri, "The SU(2) X U(1) Electroweak Model based on the Nonlinearly Realized Gauge Group. II. Functional Equations and the Weak Power-Counting," arXiv:0809.1994 [hep-th].

[3] D. Bettinelli, R. Ferrari and A. Quadri, Int. J. Mod. Phys. A 24 (2009) 2639 [arXiv:0807.3882 [hep-ph]].

[4] A. Quadri, D. Bettinelli and R. Ferrari, PoS RADCOR2007 (2007) 019. 
[5] D. Bettinelli, R. Ferrari and A. Quadri, J. General. Lie Theor. Appl. 2 (2008) 122 [arXiv:0712.1410 [hep-th]].

[6] D. Bettinelli, R. Ferrari and A. Quadri, Phys. Rev. D 77 (2008) 105012 [arXiv:0709.0644 [hep-th]].

[7] D. Bettinelli, R. Ferrari and A. Quadri, Phys. Rev. D 77 (2008) 045021 [arXiv:0705.2339 [hep-th]].

[8] D. Bettinelli, R. Ferrari and A. Quadri, "Beyond renormalization in $D=4$ : an essay on nonlinear sigma model, massive YM and Electroweak Model”, in J. M. Cornwall, A. C. Aguilar, D. Binosi and J. Papavassiliou (Eds.), Proceedings of the International Workshop on QCD Green Functions, Confinement and Phenomenology. September 7-11, 2009 ECT Trento, Italy

[9] D. Bettinelli, R. Ferrari and A. Quadri, JHEP 0703 (2007) 065 [arXiv:hep-th/0701212].

[10] D. Bettinelli, R. Ferrari and A. Quadri, Int. J. Mod. Phys. A 23 (2008) 211 [arXiv:hep-th/0701197].

[11] R. Ferrari and A. Quadri, Int. J. Theor. Phys. 45 (2006) 2497 [arXiv:hep-th/0506220].

[12] R. Ferrari and A. Quadri, JHEP 0601 (2006) 003 [arXiv:hep-th/0511032].

[13] R. Ferrari and A. Quadri, JHEP 0411 (2004) 019 [arXiv:hep-th/0408168].

[14] R. Ferrari, JHEP 0508 (2005) 048 [arXiv:hep-th/0504023]. 